Ambiente \& Água - An Interdisciplinary Journal of Applied Science
ISSN 1980-993X - doi:10.4136/1980-993X
www.ambi-agua.net
E-mail: ambi-agua@agro.unitau.br

\title{
Resíduos de pesticidas em sedimento de fundo de rio na Bacia Hidrográfica do Rio Cuiabá, Mato Grosso, Brasil
}

\author{
doi: 10.4136/ambi-agua.1263 \\ Received: 20 Dec. 2013; Accepted: 25 Feb. 2014 \\ Juliana Possavatz $^{1^{*}}$; Peter Zeilhofer ${ }^{2}$; Alicio Alves Pinto
André Luiz Tives \\ Universidade Federal de Mato Grosso - Cuiabá, MT, Brasil \\ ${ }^{1}$ Programa de Pós-Graduação em Recursos Hídricos \\ ${ }^{2}$ Departamento de Geografia \\ ${ }^{3}$ Departamento de Química \\ *Autor correspondente: e-mail: julianapossavatz@yahoo.com.br, \\ zeilhoferpeter@gmail.com, alicio_pinto@hotmail.com, andretives52@gmail.com, eliana@ufmt.br
}

\section{RESUMO}

O rio Cuiabá é um importante tributário do rio Paraguai, cuja bacia é de grande importância por agasalhar o Pantanal, a maior área úmida do planeta. A base econômica da bacia hidrográfica do rio Cuiabá é a produção agrícola e pecuária que, para manter elevada produtividade, requer grandes quantidades de insumos agrícolas como os pesticidas que, podem atingir diversos ambientes devido aos processos de dispersão que ocorrem após sua introdução no ambiente. Os sedimentos desempenham importante papel para a caracterização da poluição de rios devido a seu potencial de acumulação de poluentes. Este estudo objetivou avaliar a contaminação por pesticidas usados nas lavouras plantadas na bacia do rio Cuiabá, MT. Para tanto, amostras de sedimento de fundo foram coletadas mensalmente de agosto de 2011 a julho de 2012. Para a determinação dos pesticidas atrazina, cipermetrina, clorpirifós, endossulfam ( $\alpha, \beta$ e seu metabólito endossulfam sulfato), lambda-cialotrina, malationa, metolacloro, metribuzim, parationa metílica, permetrina e trifuralina no sedimento utilizou-se método multirresíduo que consiste na extração sólido-líquido por agitação mecânica seguida de purificação por extração líquido-líquido e identificação e quantificação por cromatografia a gás acoplada a espectrometria de massas. Resíduos de pesticidas foram identificados em amostras coletadas em agosto e setembro de 2011 e janeiro, fevereiro, abril e julho de 2012, em concentrações variando de 5,7 a 79,3 $\mu \mathrm{g} \mathrm{kg}^{-1}$. Embora a frequência de detecção tenha sido baixa, esses resultados indicam que há potencial de transporte de pesticidas usados na bacia do rio Cuiabá e seus tributários atingindo o Pantanal.

Palavras-chave: contaminação, escoamento superficial, recursos hídricos.

\section{Pesticide residue in bottom sediment in the Cuiabá River watershed, Mato Grosso, Brazil}

\section{ABSTRACT}

The Cuiabá River is a major tributary of the Paraguay River; its basin is of great importance and encloses the Pantanal, the world's largest wetland. The economic base in the 
Cuiabá River basin is agriculture and livestock production. In order to maintain high productivity, large amounts of agricultural inputs such as pesticides are required, which may reach various environments due to several dispersion processes that occur after their introduction into the environment. Sediments play an important role in the characterization of pollution of rivers and lakes due to their potential to accumulate pollutants. The present study aimed to evaluate contamination by pesticides used on crops planted in the Rio Cuiaba basin in Mato Grosso. Accordingly, samples of bottom sediment were collected monthly from August 2011 to July 2012. We used a multi-residue method, consisting of solid-liquid extraction by mechanical agitation and purification by liquid-liquid extraction. Identification and quantification was done by gas chromatography/mass spectrometry (GC/MS) for the determination of atrazine, cypermethrin, chlorpyrifos, endosulfan ( $\alpha, \beta$ and sulfate), lambdacyhalothrin, malathion, metolachlor, metribuzin, methyl parathion, permethrin and trifuralin. Pesticide residues were identified in the samples collected in August and September 2011 and in January, February, April and July 2012, at concentrations ranging from 5.7 to $79.3 \mu \mathrm{g} \mathrm{kg}^{-1}$. Although the frequency of detection was low, these results show that there is potential for the transport of pesticides used in the Cuiabá River basin and its tributaries reaching the Pantanal.

Keywords: contamination, runoff, water resources.

\section{INTRODUÇÃO}

O uso de pesticidas vem crescendo globalmente, com centenas de diferentes princípios ativos desenvolvidos para uso agrícola e não agrícola. Segundo a Agência Nacional de Vigilância Sanitária (ANVISA, 2012), o mercado brasileiro de pesticidas cresceu 190\%, ritmo muito mais acentuado do que o registrado pelo mercado mundial (93\%) entre 2000 e 2010 . De acordo com essa mesma agência, somente na safra 2010/2011 o consumo de pesticidas no Brasil atingiu a marca de 936 mil toneladas. Este elevado consumo é decorrente das grandes áreas de atividade agrícola que, no caso da região Centro-Oeste, aumentou vertiginosamente nas décadas de 70 e 80 em decorrência da ocupação dos Cerrados (Spadotto e Gomes, 2005). Contudo, estudos em várias regiões do país e do mundo evidenciam os problemas relacionados aos efeitos adversos dessas substâncias para a saúde humana e o meio ambiente (Baek et al., 2011; Cabrera et al., 2008; Delgado-Moreno et al., 2011; Malik et al., 2007; Miglioranza et al., 2013; Nogueira et al., 2012).

A bacia hidrográfica do rio Cuiabá abrange, total ou parcialmente, 29 municípios, sendo 25 localizados no Estado do Mato Grosso e 4 no Estado do Mato Grosso do Sul. O rio Cuiabá é um dos principais afluentes do rio Paraguai, cuja bacia é de grande importância nacional e internacional, por abrigar o Pantanal, uma das maiores extensões úmidas do planeta (Agência Nacional de Águas, 2003). Dentre as atividades econômicas desenvolvidas ao longo da área de drenagem dos rios Cuiabá e São Lourenço a agricultura tem papel de destaque, pois representa a base econômica da região, principalmente no cultivo da soja, algodão e milho (Mato Grosso, 2005), que vêm crescendo nos últimos 30 anos, sendo que entre os anos de 2008 e 2012, apresentou um aumento de 32,4\% (Instituto Brasileiro de Geografia e Estatística, 2013). Para manter a alta produtividade, o manejo dessas culturas requer uma elevada quantidade de insumos agrícolas, como os pesticidas que, de acordo com a maneira como são aplicados, pode sofrer diversos processos de dispersão, atingindo distintas matrizes ambientais, ameaçando assim, a sustentabilidade dos recursos hídricos da região (Dores e DeLamonica-Freire, 2001; Ribeiro et al., 2013).

Os sedimentos de fundo de rio desempenham uma importante função no processo de caracterização da poluição dos rios e lagos. A qualidade da água superficial é afetada pela 
qualidade do sedimento sobre o qual ela atravessa devido aos processos de equilíbrio que se estabelece entre água e sedimento. Além disso, os sedimentos são considerados o depósito final de muitas classes de contaminantes antropogênicos no ambiente (Jaffé, 1991, Gebremarian et al., 2012) e a dinâmica de algumas espécies químicas faz com que os sedimentos atuem como "estoque" e possíveis fontes de poluição, pois os contaminantes (espécies inorgânicas ou orgânicas) não permanentemente fixados por eles, podem ser disponibilizados para a coluna d'água devido a mudanças em parâmetros como $\mathrm{pH}$, potencial redox ou ação de microrganismos. Deste modo, podem ser importante foco de contaminantes, mesmo quando as fontes de contaminações atmosféricas e/ou terrestres já tenham sido controladas ou eliminadas (Rocha et al., 2009). Alguns trabalhos têm demonstrado que sedimentos contaminados com poluentes orgânicos persistentes tais como pesticidas organoclorados e PCBs são focos de contaminação das águas (Bradshaw et al., 2012; Granberg et al., 2008). Entretanto, a falta de dados consistentes de adsorção/dessorção dos pesticidas menos persistentes em sedimentos dificulta a previsão do comportamento destas substâncias neste compartimento (Gebremarian et al., 2012).

Considerando o intenso uso de pesticidas na bacia do rio Cuiabá, a importância desta bacia para o Pantanal e ainda a capacidade dos sedimentos de fundo de acumular contaminantes, o presente estudo teve como objetivo avaliar a contaminação por pesticidas usados nas culturas plantadas ao longo da área de drenagem do Rio Cuiabá e São Lourenço no Estado de Mato Grosso, em sedimentos de fundo dos rios. De acordo com um levantamento prévio junto ao Instituto de Defesa Agropecuária de Mato Grosso (INDEA/MT), órgão que controla as guias de comercialização, foram selecionados para determinação os seguintes princípios ativos: atrazina, metribuzim, parationa metílica, malationa, metolacloro, clorpirifós, endosulfam alfa, endosulfam beta, sulfato de endosulfam, lambda-cialotrina, permetrina e cipermetrina sendo estes identificados e quantificados por cromatografia a gás acoplada com espectrometria de massa (CG/EM).

\section{MATERIAL E MÉTODOS}

\section{1. Área de Estudo}

A bacia hidrográfica do rio Cuiabá está localizada entre as coordenadas geográficas $14^{\circ} 18^{\prime}$ e $17^{\circ} 00^{\prime}$ de latitude Sul e $54^{\circ} 40^{\prime}$ e $56^{\circ} 55^{\prime}$ de longitude Oeste, no Estado do Mato Grosso, e seus principais afluentes são os rios: Marzagão, Manso, Acorizal, Coxipó-Açú, Coxipó, Coxipó-Mirim, Aricá Açú, Aricá Mirim, Mutum e São Lourenço pela margem esquerda, e pela margem direita o Chiqueirão, Jangada, Espinheiro e Piraim (Mato Grosso, 2010).

O rio São Lourenço nasce no município de Campo Verde, banha os estados de Mato Grosso e Mato Grosso do Sul e deságua no Pantanal, sendo um dos principais afluentes da Bacia do rio Paraguai, englobando os municípios matogrossenses de Jaciara, Juscimeira, São Pedro da Cipa, Rondonópolis, Poxoréu, Dom Aquino e Campo Verde (Mato Grosso, 2008). A área de estudo no presente trabalho corresponde à bacia hidrográfica do rio Cuiabá, considerando, o rio São Lourenço como sendo uma sub-bacia do rio Cuiabá. Dessa maneira foram selecionados também para o estudo alguns dos principais afluentes do São Lourenço, são eles: o rio Tenente Amaral, Vermelho e Ponte de Pedra.

As nascentes dos rios Cuiabá e São Lourenço estão localizadas em áreas de Planalto enquanto seu exutório, no rio Paraguai, ocorre em área de planície, no Pantanal. Assim, essa bacia apresenta características tanto de rio de planalto como de planície, e as atividades desenvolvidas nas áreas mais altas podem causar impactos nas áreas baixas. 
Com relação à pluviosidade, que está fortemente relacionada ao transporte dos pesticidas pelo processo de lixiviação ou de escoamento superficial, a região caracteriza-se por possuir dois períodos bastante distintos. O período seco se estende de maio a setembro com precipitações mensais geralmente abaixo de $100 \mathrm{~mm}$, enquanto entre $70 \%$ e $80 \%$ da precipitação são concentrados no período chuvoso entre os meses de novembro a abril. Os valores anuais médios totalizam entre mais do que $2000 \mathrm{~mm}$ em algumas áreas do Planalto e menos do que $1200 \mathrm{~mm}$ na parte baixa da bacia.

As principais culturas na bacia estudada correspondem a soja, milho e algodão, que vêm crescendo nos últimos 30 anos, sendo que entre os anos de 2008 e 2012 apresentaram um aumento de $32,4 \%$, superior ao crescimento em área plantada dessas mesmas culturas registrado no país e na região Centro-Oeste (Tabela 1).

Tabela 1. Área plantada total das principais culturas nos municípios pertencentes à bacia do rio Cuiabá.

\begin{tabular}{l|cccccc}
\hline $\begin{array}{c}\text { Município/ } \\
\text { região }\end{array}$ & $\mathbf{2 0 0 8}$ & $\mathbf{2 0 0 9}$ & $\mathbf{2 0 1 0}$ & $\mathbf{2 0 1 1}$ & $\mathbf{2 0 1 2}$ & $\begin{array}{c}\text { Aumento entre } \\
\mathbf{2 0 0 8 - 2 0 1 2} \\
\mathbf{( \% )}\end{array}$ \\
\hline Brasil & 52.959 .308 & 53.557 .841 & 53.396 .971 & 56.185 .016 & 57.682 .184 & 8,92 \\
Centro-Oeste & 16.082 .019 & 16.211 .688 & 16.954 .068 & 18.166 .292 & 20.223 .292 & 25,75 \\
& 8.724 .559 & 8.649 .721 & 9.298 .438 & 9.820 .360 & 11.187 .385 & 28,22 \\
Mato-Grosso & 1.242 .318 & 1.333 .608 & 1.469 .411 & 1.374 .171 & 1.644 .849 & 32,4 \\
\hline $\begin{array}{l}\text { Bacia do rio } \\
\text { Cuiabá * }\end{array}$ & & & & & & \\
\hline
\end{tabular}

Fonte: IBGE, 2013. *valor estimado a partir do total dos 25 municípios inseridos na bacia.

\subsection{Amostragem}

Para este estudo foram selecionados nove pontos de amostragem de sedimento de fundo, conforme indicado na Figura 1 e Tabela 2, em que foram abrangidas áreas de planalto e planície. As amostras foram coletadas mensalmente entre os meses de agosto de 2011 e julho de 2012, contabilizando um total de 216 amostras. Essas amostras com massa aproximada de $1 \mathrm{~kg}$ foram coletadas usando draga de Petersen e armazenadas em sacolas plásticas previamente identificadas e transferidas para caixas de alumínio na chegada ao laboratório. Todas as amostras foram transportadas até o laboratório em caixas térmicas com gelo e armazenadas congeladas a $-20{ }^{\circ} \mathrm{C}$ até o momento das análises. A seleção dos pontos de amostragem foi feita de maneira que se tivesse uma representatividade do curso médio alto na Depressão Cuiabana, na cidade de Rosário Oeste (CBA 01), a jusante da área metropolitana de Cuiabá/Várzea Grande próximo à cidade de Santo Antônio do Leverger (CBA 02), da área de Planalto, nas cidades de Jaciara e Rondonópolis (SL 01, SL 02 e SL 03), região esta onde há a maior influência agrícola e, por último, no Pantanal, nas proximidades de Porto Cercado e em corixos, na área de planície (CBA 03, CBA 04, CBA 05 e CBA 06).

Para selecionar as moléculas a serem analisados, foi feito um levantamento dos pesticidas aplicados nos municípios pertencentes à área estudada, com base no banco de dados do Instituto de Defesa Agropecuária de Mato Grosso (INDEA/MT) referente aos anos de 2008 e 2009 (últimos dados disponíveis), a partir do qual se verificaram quais pesticidas estavam sendo utilizados em maior frequência e quantidade. 


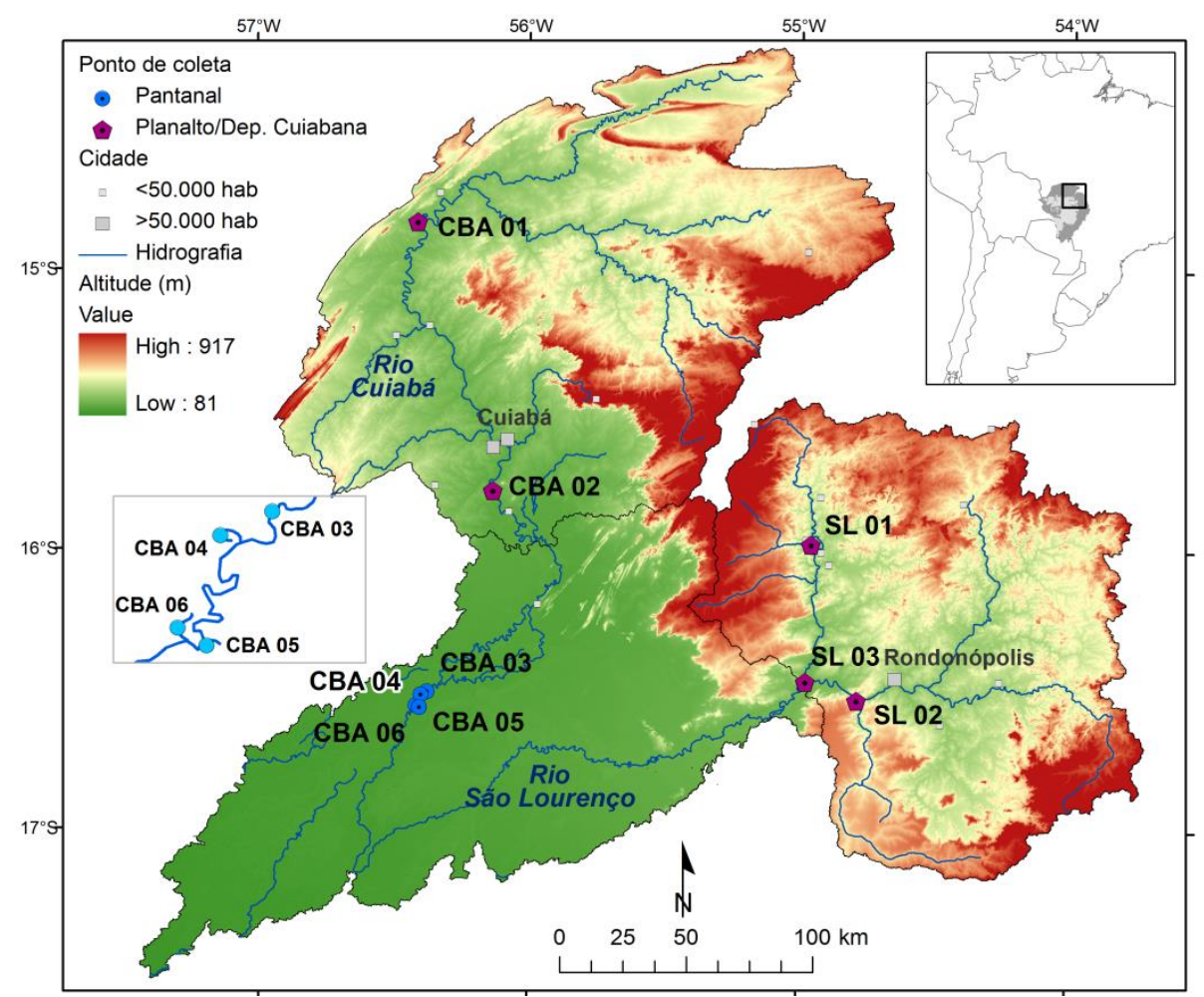

Figura 1. Mapa planialtimétrico da bacia do Rio Cuiabá com indicação dos pontos de coleta de amostras de sedimento de fundo.

Tabela 2. Coordenadas dos pontos de amostragem.

\begin{tabular}{|c|c|c|c|}
\hline Ponto & Identificação do ponto & Região & $\begin{array}{l}\text { Coordenadas } \\
\text { Geográficas }\end{array}$ \\
\hline CBA 01 & Rio Cuiabá (Ponte em Rosário Oeste) & Curso médio alto & $\begin{array}{l}\text { S } 14^{\circ} 49^{\prime} 58,9^{\prime \prime} \\
\text { W } 56^{\circ} 24^{\prime} 53,9^{\prime \prime}\end{array}$ \\
\hline CBA 02 & Rio Cuiabá (Santo Antônio) & $\begin{array}{l}\text { Curso médio } \\
\text { baixo }^{1}\end{array}$ & $\begin{array}{l}\text { S } 15^{\circ} 47^{\prime} 43,6^{\prime \prime} \\
\text { W } 56^{\circ} 07^{\prime} 38,6^{\prime \prime}\end{array}$ \\
\hline SL 01 & Rio Tenente Amaral (Jaciara) & Planalto & $\begin{array}{l}\text { S } 15^{\circ} 58^{\prime} 58,8^{\prime \prime} \\
\text { W } 54^{\circ} 57^{\prime} 17,5^{\prime \prime}\end{array}$ \\
\hline SL 02 & Rio Ponte de Pedra (Rondonópolis) & Planalto & $\begin{array}{l}\text { S } 16^{\circ} 32^{\prime} 28,6^{\prime \prime} \\
\text { W } 54^{\circ} 46^{\prime} 53,8\end{array}$ \\
\hline SL 03 & $\begin{array}{l}\text { Rio São Lourenço (Confluência do rio } \\
\text { Vermelho com o rio São Lourenço) }\end{array}$ & Planalto & $\begin{array}{l}\text { S } 16^{\circ} 28^{\prime} 36,4^{\prime \prime} \\
\text { W } 54^{\circ} 58^{\prime} 39,4^{\prime \prime}\end{array}$ \\
\hline CBA 03 & Rio Cuiabá (Porto Cercado - Pantanal) & Planície & $\begin{array}{l}\text { S } 16^{\circ} 30^{\prime} 52,7^{\prime \prime} \\
\text { W } 56^{\circ} 22^{\prime} 31,8^{\prime \prime}\end{array}$ \\
\hline CBA 04 & $\begin{array}{l}\text { Rio Cuiabá (Corixo Antonio Alves - } \\
\text { Pantanal) }\end{array}$ & Planície & $\begin{array}{l}\text { S } 16^{\circ} 31 ' 26,4^{\prime \prime} \\
\text { W } 56^{\circ} 23 ' 56,7^{\prime \prime}\end{array}$ \\
\hline CBA 05 & Rio Cuiabá (Corixo do Moquem - Pantanal) & Planície & $\begin{array}{l}\text { S } 16^{\circ} 34^{\prime} 11.0^{\prime \prime} \\
\text { W } 56^{\circ} 24^{\prime} 13,11^{\prime \prime}\end{array}$ \\
\hline CBA 06 & Rio Cuiabá (Corixão - Pantanal) & Planície & $\begin{array}{l}\text { S } 16^{\circ} 33^{\prime} 47.7^{\prime \prime} \\
\text { W } 56^{\circ} 25^{\prime} 04.3^{\prime \prime}\end{array}$ \\
\hline
\end{tabular}

Nota: ${ }^{1}$ Este ponto está localizado no início da área de planície abaixo dos dois grandes centros urbanos de Cuiabá e Várzea Grande. 


\subsection{Reagentes e Padrões}

Foram utilizados os seguintes solventes e reagentes: sulfato de sódio anidro (J. T. Baker, ACS); lã de vidro (CAAL); água ultra-purificada (Milli-Q a 18,0 M $\Omega \mathrm{cm}^{-1}$ ); n-Hexano (95\% Mallinckrodt Chemicals, para cromatografia); acetona (99,5\%, M Tedia, grau HPLC); metanol e diclorometano (99,9\%, M Tedia, grau HPLC); acetato de etila $(99,6 \%$, Mallinckrodt Chemicals); tolueno (99,8\%, Mallinckrodt Chemicals, grau HPLC). Os padrões analíticos de alta pureza (> 95,4\%) dos pesticidas estudados (atrazina, cipermetrina, clorpirifós, endosulfam ( $\alpha, \beta$ e sulfato), lambda cialotrina, malationa, metolacloro, metribuzim, parationa metílica, permetrina e trifuralina) e do padrão interno fenantreno D-10, foram adquiridos das empresas Dr. Ehrenstorfer Gmbh (Augsburg, Alemanha), SigmaAldrich Laborchemikalien GmbH (Riedel-de-Haen) (Seelze, Alemanha) e Sigma-Aldrich Chemie GmbH (Steinheim, Alemanha).

\subsection{Método de extração de resíduos de pesticidas em sedimento por CG/EM}

A extração dos pesticidas das amostras de sedimento de fundo foi realizada seguindo o procedimento descrito por Laabs et al. (2002), otimizado por Miranda (2008). Para tanto, pesaram-se 20,0 g de amostra úmida em Erlenmeyer, aos quais foram adicionados $50 \mathrm{~mL}$ da mistura de solventes acetona, acetato de etila e água, na proporção de 2:2:1 (v/v/v) e submetido à agitação mecânica por 4 horas. Em seguida, o extrato foi filtrado usando papel de filtro faixa média e concentrado em evaporador rotatório a $42{ }^{\circ} \mathrm{C}$, rotação de $90 \mathrm{rpm}$ e pressão de 300 mbar. Após evaporação da fase orgânica, foram adicionados $30 \mathrm{~mL}$ de solução saturada de cloreto de sódio ao extrato concentrado, o qual foi submetido a extração líquidolíquido com diclorometano ( $1 \times 30 \mathrm{~mL}$ mais $2 \times 25 \mathrm{~mL})$. A fase contendo diclorometano foi filtrada em funil de vidro, contendo sulfato de sódio anidro e lã de vidro, sendo posteriormente purificado com alumina e florisil.

Para a purificação condicionou-se, com hexano, $1,0 \mathrm{~g}$ de alumina e 1,0 $\mathrm{g}$ de florisil empacotados em cartucho de vidro, ativando-se as fases, previamente, por meio de aquecimento em mufla a $600{ }^{\circ} \mathrm{C}$ durante 4 horas, sendo em seguida adicionados $0,06 \mathrm{~g}$ e $0,1 \mathrm{~g}$ de água deionizada para cada $1,0 \mathrm{~g}$ de fase, respectivamente. Após a transferência do extrato para a coluna, os pesticidas foram eluídos com $10 \mathrm{~mL}$ de hexano e $10 \mathrm{~mL}$ da mistura de solventes, hexano e acetato de etila, na proporção de 4:1 (v/v). O eluato foi concentrado em evaporador rotatório $\left(45^{\circ} \mathrm{C}, 80 \mathrm{rpm}\right.$ e 400 mbar) e retomado com alíquotas de tolueno em frasco de autoamostrador (vials) contendo $100 \mu \mathrm{L}$ do padrão interno fenantreno D-10 $\left(1 \mu \mathrm{g} \mathrm{mL}{ }^{-1}\right)$ e armazenado em refrigerador $\left(-4^{\circ} \mathrm{C}\right)$ até análise por CG/EM.

Os procedimentos de extração foram feitos em duplicata e os resultados $\left(\mu \mathrm{g} \mathrm{kg}^{-1}\right.$ de peso seco) apresentados como a média dos valores determinados. A determinação do peso seco das amostras foi realizada por gravimetria para correção do resultado final. A cada lote de amostras adicionava-se uma amostra controle (isenta da matriz) visando controlar qualquer possível interferente decorrente do procedimento de extração.

\subsection{Condições Cromatográficas}

Para a separação e identificação dos analitos foi utilizado um cromatógrafo a gás (modelo HP 6890) com injetor automático (modelo 7683B series), acoplado a um espectrômetro de massa (modelo HP 5973), equipado com coluna HP 19091-433 com fase HP-5 (5\% de fenil metil siloxano, $30,0 \mathrm{~m} \times 250 \mu \mathrm{m}$ d.i. $\times 0,25 \mu \mathrm{m}$ de espessura do filme). Para a separação adequada dos analitos no sistema $\mathrm{CG} / \mathrm{EM}$, foi utilizada a seguinte programação otimizada de temperatura do forno: temperatura inicial de $92^{\circ} \mathrm{C}$ mantida por $2,5 \mathrm{~min}$, em seguida rampa de $15^{\circ} \mathrm{C} \mathrm{m^{-1 }}$ até $175^{\circ} \mathrm{C}$ mantida por $13 \mathrm{~min}$, e rampa de $20^{\circ} \mathrm{C} \mathrm{min}{ }^{-1}$ até $280{ }^{\circ} \mathrm{C}$ e mantida por $15 \mathrm{~min}$. As demais condições do método de análise foram: volume de injeção de 1,0 $\mu \mathrm{L}$ (sem divisão de fluxo), fluxo do gás de arraste (He, pureza 99,9999\%) igual a 1,0 mL min ${ }^{-1}$, 
ionização por impacto eletrônico de $70 \mathrm{eV}$, temperatura da fonte de ionização de $230^{\circ} \mathrm{C}$, do quadrupolo de $150^{\circ} \mathrm{C}$, da linha de transferência de $280^{\circ} \mathrm{C}$ e do injetor de $250^{\circ} \mathrm{C}$. O método do padrão interno foi utilizado para a quantificação em modo monitoramento de íon selecionado (SIM), sendo o íon principal utilizado para a quantificação e os íons secundários para a identificação (Tabela 3). Para confirmação da identidade dos pesticidas detectados, foi considerado um máximo de $20 \%$ de diferença entre a abundância relativa esperada dos íons secundários em relação ao principal.

Tabela 3. Pesticidas determinados, íons correspondentes monitorados e limites de detecção e quantificação dos métodos que emprega CG/EM-SIM.

\begin{tabular}{|c|c|c|c|c|c|c|c|}
\hline Pesticidas & $\begin{array}{c}\operatorname{Tr}^{2} \\
(\mathbf{m i n})\end{array}$ & $\begin{array}{c}\mathbf{L D}^{1} \\
\left(\mu \mathbf{g ~ k g}^{-1}\right)\end{array}$ & $\begin{array}{c}\mathbf{L Q}^{1} \\
\left(\mu \mathrm{g} \mathrm{kg}^{-1}\right)\end{array}$ & Recuperação $^{1}$ & $\begin{array}{c}\text { Íon } \\
\text { Principal }\end{array}$ & $\begin{array}{c}\text { Secundário } \\
1\end{array}$ & $\begin{array}{c}\text { Secundário } \\
2\end{array}$ \\
\hline Atrazina & 12,8 & 1,0 & 7,0 & 92 & 200,1 & 202,1 & 215 \\
\hline Cipermetrina & 29,4 & 2,5 & 6,0 & 109 & 181,1 & 163,0 & 165,0 \\
\hline$\lambda$-cialotrina & 27,6 & 1,0 & 5,0 & 86 & 181,0 & 197,0 & 208,0 \\
\hline Clorpirifós & 21,4 & 1,0 & 6,0 & 85 & 198,9 & 196,9 & 313,9 \\
\hline$\alpha$-endossulfam & 24,0 & 1,0 & 5,0 & 95 & 240,9 & 238,9 & 242,9 \\
\hline$\beta$-endossulfam & 25,2 & 5,0 & 6,0 & 103 & 236,8 & 238,8 & 195,0 \\
\hline $\begin{array}{l}\text { Endossulfam } \\
\text { sulfato }\end{array}$ & 25,9 & 2,5 & 30,0 & 95 & 271,8 & 273,8 & 236,9 \\
\hline Malationa & 20,7 & 2,0 & 31,0 & 76 & 125,0 & 127,0 & 173,1 \\
\hline Metolacloro & 20,9 & 1,0 & 7,0 & 89 & 162,1 & 238,1 & 240,1 \\
\hline Metribuzina & 16,5 & 2,5 & 5,0 & 77 & 198,0 & 199,0 & 182,0 \\
\hline $\begin{array}{l}\text { Parationa } \\
\text { Metílica }\end{array}$ & 17,0 & 2,5 & 29,0 & 78 & 109,0 & 125,0 & 263,0 \\
\hline Permetrina & 28,3 & 1,0 & 7,0 & 98 & 163,0 & 183,0 & 165,0 \\
\hline Trifuralina & 11,3 & 1,0 & 6,0 & 88 & 264,0 & 290,0 & 306,0 \\
\hline $\begin{array}{l}\text { Fenantreno D- } \\
10^{3}\end{array}$ & 13,5 & & & & 184,1 & 188,1 & 189,1 \\
\hline
\end{tabular}

Fonte: ${ }^{\mathbf{1}}$ Miranda (2008). ${ }^{2} \mathrm{Tr}=$ tempo de retenção; ${ }^{3}$ Padrão interno.

\section{RESULTADOS E DISCUSSÃO}

A partir dos dados de utilização de pesticidas nos municípios que compõem a bacia hidrográfica do rio Cuiabá, identificou-se que os mais utilizados foram acefato, atrazina, carbofurano, cardosulfano, clomazona, clorpirifós, diurom, endossulfam, glifosato, metomil, parationa metílica, permetrina e tebuconazol. Dentre estes, foram selecionados para determinação os pesticidas relacionados na Tabela 4, cujo potencial de transporte por 
escoamento superficial, dissolvidas em água ou associadas ao sedimento foi avaliado utilizando os critérios publicados por Goss (1992).

Tabela 4. Potencial de transporte superficial das moléculas estudadas conforme critérios reportados por Goss (1992).

\begin{tabular}{|c|c|}
\hline Alto potencial de transporte dissolvido em água & Pesticidas \\
\hline $\begin{array}{l}\mathrm{t}_{1 / 2} \text { no solo }>35 \text { dias } ; \mathrm{K}_{\mathrm{oc}}<100000 ; \mathrm{S}_{\mathrm{w}} \geq 1 \mathrm{mg} \mathrm{L}^{-1} \\
\text { ouK }_{\mathrm{oc}} \leq 700 \text { e } 10 \leq \mathrm{S}_{\mathrm{w}} \leq 100 \mathrm{mg} \mathrm{L}^{-1}\end{array}$ & $\begin{array}{l}\text { atrazina } \\
\text { clorpirifós } \\
\text { metolacloro } \\
\text { parationa metílica }\end{array}$ \\
\hline Baixo potencial de transporte dissolvido em água & Pesticidas \\
\hline $\begin{array}{l}\mathrm{K}_{\mathrm{oc}} \geq 100000 \text { ou } \mathrm{t}_{1 / 2} \text { no solo } \leq 1 \text { dia ou } \\
\mathrm{K}_{\mathrm{oc}} \leq 100 ; \mathrm{t}_{1 / 2} \text { no solo }<35 \text { dias; } \mathrm{S}_{\mathrm{w}}<0,5 \mathrm{mg} \mathrm{L}^{-1}\end{array}$ & $\begin{array}{l}\lambda \text {-cialotrina } \\
\text { malationa }\end{array}$ \\
\hline Alto potencial de transporte associado ao sedimento & Pesticidas \\
\hline $\begin{array}{l}\mathrm{t}_{1 / 2} \text { no solo } \geq 40 \text { dias } ; \mathrm{K}_{\mathrm{oc}}=1000 \text { ou } \\
\mathrm{t}_{1 / 2} \text { no solo } \geq 40 \text { dias; } \mathrm{K}_{\mathrm{oc}} \geq 500 ; \mathrm{S}_{\mathrm{w}}=0,5 \mathrm{mg} \mathrm{L}^{-1}\end{array}$ & $\begin{array}{l}\text { cipermetrina } \\
\text { clorpirifós } \\
\text { endossulfam } \\
\lambda \text {-cialotrina } \\
\end{array}$ \\
\hline Baixo potencial de transporte associado ao sedimento & Pesticidas \\
\hline $\begin{array}{l}\mathrm{t}_{1 / 2} \text { no solo }<1 \text { dia ou } \leq 40 \text { dias; } \mathrm{K}_{\mathrm{oc}} \leq 500 ; \mathrm{S}_{\mathrm{w}} \geq 0,5 \mathrm{mg} \mathrm{L}^{-1} \\
\text { ou } \mathrm{t}_{1 / 2} \text { no solo } \leq 2 \text { dias ou } \mathrm{t}_{1 / 2} \text { no solo } \leq 4 \text { dias; } \mathrm{K}_{\mathrm{oc}} \leq 900 ; \mathrm{S}_{\mathrm{w}} \geq 0,5 \mathrm{mg} \mathrm{L}^{-1} \\
\text { ou } \mathrm{t}_{1 / 2} \text { no solo } \leq 40 \text { dias; } \mathrm{K}_{\mathrm{oc}} \leq 900 ; \mathrm{S}_{\mathrm{w}} \geq 2,0 \mathrm{mg} \mathrm{L}^{-1}\end{array}$ & $\begin{array}{l}\text { metribuzim } \\
\text { parationa metílica }\end{array}$ \\
\hline
\end{tabular}

Como já visto na Tabela 1, a área total plantada com culturas agrícolas na bacia aumentou em 32,4\% nos últimos quatro anos, fator este que demanda um aumento também na quantidade de pesticidas aplicados nos municípios formadores da bacia. Embora novos princípios ativos possam ter surgido desde 2009 e dados atualizados sobre o uso de pesticidas não estejam disponíveis, verificou-se que os tipos de culturas de maior predominância não variaram e, dessa forma, a grande maioria dos princípios ativos utilizados possivelmente continuam sendo os mesmos. Algumas culturas agrícolas merecem atenção, não pela quantidade de pesticidas aplicados por área cultivada, e sim por essas culturas ocuparem extensas áreas nos municípios da bacia, como é o caso da soja, do milho e do algodão (Instituto Brasileiro de Geografia e Estatística, 2013).

Durante os 12 meses de monitoramento (agosto de 2011 a julho de 2012), verificou-se a presença de resíduos de pesticidas em apenas algumas amostras coletadas nos meses de agosto e setembro do ano de 2011 e nos meses de janeiro, fevereiro, abril e julho de 2012, sendo detectadas concentrações variando de 5,7 a 79,3 $\mathrm{g} \mathrm{kg}^{-1}$ (Tabela 5). Os pesticidas detectados são usados com frequência na agricultura e sua presença no sedimento deve-se provavelmente ao seu transporte adsorvido ao material particulado através do escoamento superficial.

Apresentaram contaminação amostras coletadas no ponto CBA 01 localizado no curso médio alto no município de Rosário Oeste, mais próximo da região de nascente do Rio Cuiabá, nos pontos situados em área de Planalto no Rio Tenente Amaral, afluente do rio São Lourenço em Jaciara (SL 01), bem como no encontro dos rios Vermelho e São Lourenço (SL 03); além de amostras coletadas em área de planície, em pontos no rio Cuiabá no Pantanal em Porto Cercado (CBA 03) e nos pontos correspondentes aos corixos Antônio Alves (CBA 04) e do Moquem (CBA 05). 
Dos pesticidas detectados, quatro deles ( $\alpha$-endossulfam, endossulfam sulfato, $\lambda$-cialotrina e clorpirifós), de acordo com a classificação de Goss (1992), apresentam alto potencial de transporte associado ao sedimento destacando ainda o clorpirifós, que pode ser transportado também dissolvido em água. Isso indica que esses compostos atingiram os corpos d'águas através do escoamento superficial estando fortemente ligados ou sorvidos nas moléculas do solo. Já o metolacloro e a malationa apresentam maior possibilidade de ter atingido os recursos hídricos superficiais dissolvidos em água no processo de escoamento superficial e embora essas moléculas não tenham sido detectados no período de chuva sua presença pode estar associada a eventos de chuva anteriores, uma vez que a presença no sedimento pode não refletir uma contaminação atual (Tabela 4 ).

Tabela 5. Concentrações de pesticidas detectadas em sedimento de fundo ( $\mu \mathrm{g} \mathrm{kg}^{-1}$ de peso seco), por ponto de coleta.

\begin{tabular}{l|ccc|cc|cc|c|c|c}
\hline \multirow{2}{*}{ Pesticidas } & \multicolumn{3}{|c|}{$\begin{array}{c}\text { Ago/11 } \\
\text { (seca) }\end{array}$} & \multicolumn{2}{c|}{$\begin{array}{c}\text { Set/11 } \\
\text { (seca) }\end{array}$} & \multicolumn{2}{c|}{$\begin{array}{c}\text { Jan/12 } \\
\text { (chuvoso) }\end{array}$} & $\begin{array}{c}\text { Fev/12 } \\
\text { (chuvoso) }\end{array}$ & $\begin{array}{c}\text { Abr/12 } \\
\text { (chuvoso) }\end{array}$ & $\begin{array}{c}\text { Jul/12 } \\
\text { (seca) }\end{array}$ \\
\cline { 2 - 10 } & CBA 01 & SL 03 & CBA 03 & SL 01 & SL 03 & CBA & SL 01 & CBA 05 & CBA 04 & CBA 01 \\
\hline Metolacloro & -2 & 21,2 & 19,1 & - & - & - & - & - & - & - \\
Permetrina & 44,5 & - & - & - & - & - & - & - & - & - \\
$\lambda$-cialotrina & 33,8 & - & 60,0 & 19,7 & 31,0 & - & - & - & - & - \\
$\alpha$-endossulfam & - & - & - & - & - & - & 13,6 & - & - & - \\
Endo. Sulfato & - & - & - & - & - & 5,7 & 25,9 & 79,3 & - & - \\
Clorpirifós & - & - & - & - & - & - & - & - & - & 8,5 \\
Malationa & - & - & - & - & - & - & - & - & 9,2 & - \\
\hline
\end{tabular}

Nota: ${ }^{1}$ período de seca e chuvoso característico do mês de coleta indicado entre parêntesis; ${ }^{2}-<$ LD do método; ${ }^{3}$ endossulfam sulfato (principal metabólito do endossulfam).

Embora dados atualizados de pesticidas utilizados na área de estudo não puderam ser disponibilizados, usando os dados de uso de pesticidas na bacia dos anos de 2008 e 2009, observou-se que dentre os pesticidas detectados em sedimento de fundo, o clorpirifós e endossulfam vinham sendo aplicados nos municípios onde foram detectados, ressaltando que ambas as moléculas apresentam persistência considerável, com tempos de meia-vida no solo iguais a 50 dias.

Desde a década de 70, a expansão da pecuária e da agricultura em áreas do Planalto tem aumentado o desmatamento e a erosão, e vários rios da região, como o Taquari e o São Lourenço, apresentam elevada capacidade de transporte de sedimentos. Isto tem aumentado a deposição de sedimentos no Pantanal e o consequente assoreamento dos rios localizados nas regiões de menor altitude (Agência Nacional de Águas, 2013). Os resultados obtidos no presente estudo indicam que pesticidas utilizados na bacia do Rio Cuiabá e em seus afluentes podem atingir o Pantanal (presença de endossulfam sulfato, clorpirifós, malationa e $\lambda$ cialotrina em pontos de coleta na planície), sendo transportados adsorvidos ao material particulado. Uma vez que as configurações geomorfológica e climatológica dentro da bacia fazem com que o escoamento proveniente do planalto seja direcionado diretamente para o Pantanal, as ações produzidas na parte superior da bacia (planalto) podem ocasionar impactos diretos sobre o Pantanal e áreas à jusante. Entretanto, devido à sua ocorrência eventual e com 
baixa frequência ao longo da bacia, não há indicação de um elevado risco de acumulação na planície.

Não foi possível verificar influência da época de coleta nos resultados. Tal fato deve-se provavelmente à característica difusa da contaminação por pesticidas e à dinâmica de sedimentação que varia durante as estações. Soma-se a isto a eventual degradação das moléculas tanto no sedimento como na coluna d'água. Não se pode deixar de considerar também que as concentrações de pesticidas encontradas no ambiente estão em geral muito próximas dos limites de detecção dos métodos analíticos e caso ocorra uma pequena diluição as concentrações podem atingir valores não detectáveis.

Em estudo desenvolvido por Laabs et al. (2002) na região nordeste do Pantanal, no qual foram coletadas amostras de sedimento de fundo nos rios São Lourenço e Cuiabá e na Baía de Siá Mariana na planície pantaneira, foram detectados ametrina, p,p'DDT, p,p'DDE, desetilatrazina, $\beta$-endossulfam, sulfato de endossulfam e simazina em concentrações variando de 0,4 a $4,5 \mu \mathrm{g} \mathrm{kg}^{-1}$, valores estes inferiores aos detectados no presente estudo. Aqueles autores coletaram amostras de novembro de 1999 a março de 2000, ou seja, somente no período chuvoso, época em que, apesar dos processos de transporte superficial serem intensificados, há também maior vazão nos rios e portanto menos deposição. Por outro lado, na área de planície os autores encontraram somente os organoclorados, mais persistentes, sendo os demais pesticidas detectados somente no planalto. No presente estudo, os pesticidas foram detectados com mais frequência nos pontos localizados no planalto, apesar de terem sido encontrados também nas amostras coletadas na planície.

Cunha (2003) avaliou o nível de contaminação de 37 pesticidas no sedimento de rio, coletados nos principais rios às bordas do Pantanal. A amostragem foi feita em duas épocas, novembro de 2001 e fevereiro de 2002, que corresponde ao início das chuvas e final das chuvas respectivamente. Este autor detectou alacloro, ametrina, clorpirifós, p,p'DDE, p,p'DDT, endossulfam sulfato, beta endossulfam, metolacloro, metoxicloro, metribuzim, simazina, terbutilazina, trifluralina. Dos princípios ativos detectados por Cunha (2003), da mesma forma, o metolacloro, o endossulfam sulfato e o clorpirifós foram também detectados em amostras de sedimento de fundo de rio avaliadas no presente estudo.

Miranda et al. (2008) verificaram a contaminação por resíduos de pesticidas nos sedimentos de fundo de 17 rios formadores do Pantanal, dentre eles, o Cuiabá, o São Lourenço e o Vermelho, que também foram avaliados no presente trabalho. Os compostos identificados em amostras de sedimento incluíram $\lambda$-cialotrina $\left(1,0-5,0 \mu \mathrm{g} \mathrm{kg}^{-1}\right)$, p,p'DDT $(3,6$ $\left.\mu \mathrm{g} \mathrm{kg}{ }^{-1}\right)$, deltametrina $\left(20,0 \mu \mathrm{g} \mathrm{kg}^{-1}\right)$ e permetrina $\left(1,0-7,0 \mu \mathrm{g} \mathrm{kg}^{-1}\right)$. Os princípios ativos $\lambda$ cialotrina e permetrina também foram avaliados no presente estudo, entretanto, somente a $\lambda$ cialotrina foi identificada em amostras de sedimento de fundo de rio apresentando concentrações superiores àquelas verificadas por Miranda.

Inseticidas piretróides têm sido detectados em sedimentos de fundo de rio em diversos países do mundo (Delgado-Moreno et al., 2011; Ensminger et al., 2013; Li et al., 2013; Toan et al., 2013) com destaque para a cipermetrina, deltametrina, bifentrina, $\lambda$-cialotrina e permetrina, sendo estas duas últimas detectadas no presente trabalho. Tal fato deve-se provavelmente ao uso intensivo dessa classe de pesticidas em diversas culturas associado aos elevados coeficientes de sorção ( $\mathrm{K}_{\mathrm{oc}}$ entre 100.000 e $1.000 .000 \mathrm{~mL} \mathrm{~g}^{-1}$ ) destas moléculas ao solo que contribui para seu carreamento para os cursos d'água associados ao particulado.

Outros pesticidas detectados com frequência em sedimento por vários autores em diferentes regiões do mundo são o endossulfam (isômeros alfa e beta e seu principal metabólito sulfato de endossulfam) e o clorpirifós (Masiá et al., 2013; Miglioranza et al., 2013; Li et al., 2013; Silva et al., 2008). Em estudo realizado no Pantanal por Laabs et al. (2007) no qual foi avaliada a dissipação de nove pesticidas usando mesocosmos, os autores observaram que o endossulfam beta seguido do metolacloro, atrazina, simazina e clorpirifós 
foram os mais persistentes nestes ambientes sendo que o endossulfam e o clorpirifós tenderam a se acumular no sedimento, o que explica a detecção dessas moléculas nas amostras analisadas.

\section{CONCLUSÃO}

De um total de 216 amostras, apenas 10 apresentaram contaminação com pelo menos um princípio ativo, em concentrações variando de 5,7 a 79,3 $\mu \mathrm{g} \mathrm{kg}^{-1}$, sendo detectados clorpirifós, endossulfam ( $\alpha$ e sulfato), $\lambda$-cialotrina, malationa, metolacloro e permetrina. Embora a frequência de detecção tenha sido baixa, verificou-se a presença de resíduos de pesticidas em amostras correspondentes ao Pantanal. Conclui-se assim que há potencial para transporte de pesticidas para dentro do ambiente aquático na bacia do Rio Cuiabá mesmo que em concentrações e frequência de detecção baixas.

\section{REFERÊNCIAS}

AGÊNCIA NACIONAL DE ÁGUAS - ANA (Brasil). Projeto de implementação de práticas de gerenciamento integrado de bacia hidrográfica para o Pantanal e Alto Paraguai. ANA/GEF/PNUMA/OEA: Subprojeto: avaliação dos mecanismos financeiros para gerenciamento sustentável da bacia piloto do Rio Cuiabá. Brasília, DF, 2003.

AGÊNCIA NACIONAL DE ÁGUAS - ANA (Brasil). Região hidrográfica do Paraguai. Brasília, DF, 2013. Disponível em: <http://www2.ana.gov.br/Paginas/portais /bacias/paraguai.aspx>. Acesso em: $10 \mathrm{dez} .2013$.

AGÊNCIA NACIONAL DE VIGILÂNCIA SANITÁRIA - ANVISA (Brasil). Seminário volta a discutir mercado de agrotóxicos em 2012. Brasília, DF, 2012. Disponível em: $<$ http://portal.anvisa.gov.br/wps/content/anvisa+portal/anvisa/sala+de+imprensa/menu+ +noticias+anos/2012+noticias/seminario+volta+a+discutir+mercado+de+agrotoxicos+e m+2012>. Acesso em: 12 dez. 2013.

BAEK, S.; CHOI, S.; CHANG, Y. Three-year atmospheric monitoring of organochlorine pesticides and polychlorinated biphenyls in polar regions and the south pacific. Environmental Science and Technology, v. 45, n. 10, p. 4475-4482, 2011. http://dx.doi.org/10.1021/es1042996

BRADSHAW C.; TJENSVOLL, I.; SKÖLD, M.; ALLAN, I. J.; MOLVAER, J.; MAGNUSSON, J.; NAES, K.; NILSSON, H. C. Bottom trawling resuspends sediment and releases bioavailable contaminants in a polluted fjord. Environmental Pollution, v. 170, p. 232-241, 2012. http://dx.doi.org/10.1016/j.envpol.2012.06.019

CABRERA, C. L; COSTA, F. B; PRIMEL, E. G. Estimativa de risco de contaminação das águas por pesticidas na região sul do estado do RS. Química Nova, v. 31, n. 8, p.19821986, 2008. http://dx.doi.org/10.1590/S0100-40422008000800012

CUNHA, M. L. Determinação de resíduos de pesticidas em sedimentos dos principais rios do Pantanal Mato-grossense por CG/EM. 2003. 91f. Dissertação (Mestrado em Saúde e Ambiente) - Instituto de Saúde Coletiva, Universidade Federal de Mato Grosso, Cuiabá, 2003. 
DELGADO-MORENO, L.; LIN, K.; VEIGA-NASCIMENTO, R.; GAN, J. Occurrence and toxicity of three classes of insecticides in water and sediment in two Southern California Coastal Watersheds. Journal of Agricultural and Food Chemistry, v. 59, p. 94489456, 2011. http://dx.doi.org/10.1021/jf202049s

DORES, E. F. G. C.; DE-LAMONICA-FREIRE, E. M. Contaminação do ambiente aquático por pesticidas: estudo de caso: águas usadas para consumo humano em Primavera do Leste, Mato Grosso - Análise preliminar. Química Nova, v. 24, n. 1, p. 27-36, 2001. http://dx.doi.org/10.1590/S0100-40422001000100007

ENSMINGER, M. P.; BUDD, R.; KELLEY, K. C.; GOH, K. S. Pesticide occurrence and aquatic benchmark exceedances in urban surface waters and sediments in three urban areas of California, USA, 2008-2011. Environmental Monitoring and Assessment, v. 185, p. 3697-3710, 2013. http://dx.doi.org/10.1007/s10661-012-2821-8

GEBREMARIAM, S. Y; BEUTEL, M. W.; YONGE, D.R.; FLURY, M.; HARSH, J. B. Adsorption and desorption of chlorpyrifos to soils and sediments. Reviews of Environmental Contamination and Toxicology, v. 215, p. 123-75, 2012. http://dx.doi.org/10.1007/978-1-4614-1463-6_3

GOSS, D. W. Screening procedure for soils and pesticides potential water quality impacts. Weed Technology, v. 6, p. 701-708, 1992. http://www.jstor.org/stable/3987238

GRANBERG , M. E.; GUNNARSSON , J. S.; HEDMAN , J. E.; ROSENBERG , R.; JONSSON , P. Bioturbation-driven release of organic contaminants from Baltic Sea sediments mediated by the invading polychaete Marenzelleria neglecta. Environmental Science and Technology, v. 42, p. 1058-1065, 2008. http://dx.doi.org/10.1021/es071607j

INSTITUTO BRASILEIRO DE GEOGRAFIA E ESTATÍSTICA - IBGE. Relatório de área plantada por município no Mato Grosso, 2008-2012. Rio de Janeiro, 2013.

JAFFÉ, R. Fate of hydrophobic organic pollutants in the aquatic environment: a review. Environmental Pollutions, v. 69, p. 237-257, 1991. http://dx.doi.org/10.1016/02697491(91)90147-O

LAABS, V.; AMElUNG, W.; PINTO, A.; WANTZEN, M.; SILVA, C. J.; ZECH, W. Pesticides in surface water, sediment, and rainfall of the Northeastern Pantanal Basin, Brazil. Journal of Environmental Quality, v. 31, p. 1636-1648, 2002. http://dx.doi.org/10.2134/jeq 2002.1636

LAABS, W.; WEHRHAN, A.; PINTO, A. A.; DORES, E. F. G. C.; AMELUNG, W. Pesticide fate in tropical wetlands of Brazil: An aquatic microcosm study under semifield conditions. Chemosphere, v. 67, p. 975-989, 2007. http://dx.doi.org/10.1016/j. chemosphere.2006.10.067

LI, H.; SUN, B.; LYDY, M. J.; YOU, J. Sediment-associated pesticides in an urban stream in Guangzhou, China: implication of a shift in pesticide use patterns. Environmental Toxicology and Chemistry, v. 32, n. 5, p. 1040-1047, 2013. http://dx.doi.org/10.1002/etc.2147

MALIK, A.; SINGH, V. D. K.; SINGH, K. P. Occurrence and distribution of persistent trace organics in rainwater in an urban region (India). Bulletin of Environmental

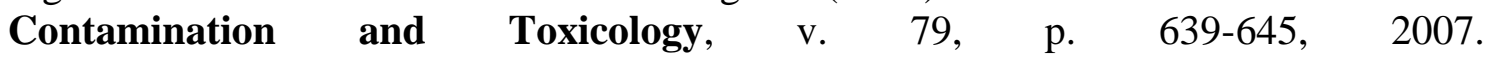
http://dx.doi.org/10.1007/s00128-007-9290-8 
MASIÁ, A.; CAMPO, J.; VÁZQUEZ-ROIG, P.; BLASCO, C.; PICÓ, Y. Screening of currently used pesticides in water, sediments and biota of the Guadalquivir River Basin (Spain). Journal of Hazardous Materials, v. 263P, p. 95-104, 2013. http://dx.doi.org/10.1016/j.jhazmat.2013.09.035

MATO GROSSO. Secretaria de Estado do Meio Ambiente. Superintendência de monitoramento de indicadores ambientais. Relatório de Monitoramento de Qualidade das Águas da Sub-bacia do Rio Cuiabá, MT - 2005. Cuiabá: SEMA/MT; SMIA, 2006.

MATO GROSSO. Secretaria de Estado do Meio Ambiente. Recuperação de áreas degradadas: uma proposta para o cerrado da Bacia Hidrográfica do Rio São Lourenço - Mato Grosso. Brasília, 2008. Disponível em: <http://lcf.esalq.usp.br/prof/pedro/lib/exe/fetch.php?media= ensino:graduacao:manual_2008_recuperacao_bacia_do_sao_lourenco.pdf.> Acesso em: 12 Dez. 2013.

MATO GROSSO. Secretaria de Estado do Meio Ambiente. Superintendência de Monitoramento de Indicadores Ambientais. Relatório de monitoramento da qualidade da água da região hidrográfica do Paraguai - 2007 a 2009. Cuiabá: SEMA/MT; SMIA, 2010.

MIGLIORANZA, K. S. B.; GONZALEZ, M.; ONDARZA, P. M.; SHIMABUKURO, V. M.; ISLA, F. I.; FILLMANN, G. et al. Assessment of Argentinean Patagonia pollution: PBDEs, OCPs and PCBs in different matrices from the Río Negro basin. Science of the Total Environment, v. 452-453, p. 275-285, 2013. http://dx.doi.org/10.1016/j. scitotenv.2013.02.055

MIRANDA, K. A.; CUNHA, M. L. F.; DORES, E. F. G. C.; CALHEIROS, D. F. Pesticide residues in river sediments from the Pantanal Wetland, Brazil. Journal of Environmental Science and Health B, v. 43, p. 717-722, 2008. http://dx.doi.org/10.1080/03601230802388843

NOGUEIRA, E. N.; DORES, E. F. G. C.; PINTO, A. A.; AMORIM, R. S. S.; RIBEIRO, M. L.; LOURENCETTI, C. Currently used pesticides in water matrices in central-western Brazil. Journal of Brazilian Chemical Society, v. 23, p. 1476-1487, 2012. http://dx.doi.org/10.1590/S0103-50532012005000008

RIBEIRO, A. C. A.; DORES, E. F. G. C.; AMORIM, R. S. S.; LOURENCETTI, C. Resíduos de pesticidas em águas superficiais de área de nascente do rio São Lourenço-MT: Validação de método por extração em fase sólida e cromatografia líquida. Química Nova, p. 284-290, 2013. http://dx.doi.org/10.1590/S0100-40422013000200015

ROCHA, J. C.; ROSA, A. H.; CARDOSO A. A. Introdução à química ambiental. 2. ed. São Paulo: Artmed 2009. p. 29.

SILVA, D. M. L.; CAMARGO, P. B.; MARTINELlI, L. A.; LANÇAS, F. M.; PINTO, J. S. S.; AVELAR, W. E. P. Organochlorine pesticides in Piracicaba river basin (São Paulo/Brazil): a survey of sediment, bivalve and fish. Química Nova, v. 31, n. 2, p. 214-219, 2008. http://dx.doi.org/10.1590/S0100-40422008000200005

SPADOTTO, C. A.; GOMES, M. A. F. Agrotóxicos no Brasil. Brasília, DF: EMBRAPA, 2005. Disponível em: <http://www.agencia.cnptia.embrapa.br/gestor/agricultura_e_ meio_ambiente/arvore/CONTAG01_40_210200792814.html>. Acesso em: 12 dez. 2013. 
TOAN, P. V.; SEBESVARI, Z.; BLÄSING, M.; ROSENDAHL, I.; RENAUD, F. G. Pesticide management and their residues in sediments and surface and drinking water in the Mekong Delta, Vietnam. Science of the Total Environment, v. 452-453, p. 28-39, 2013. http://dx.doi.org/10.1016/j.scitotenv.2013.02.026 